\title{
Estimation of the vitrified canister production for a PWR fleet with the CLASS code
}

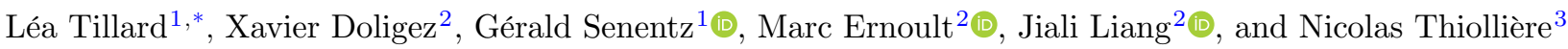 \\ ${ }^{1}$ Orano, 92320 Châtillon, France \\ 2 IJCLab, IN2P3-CNRS, Université Paris-Saclay, 91406 Orsay Cedex, France \\ ${ }^{3}$ Laboratoire Subatech, IMTA-IN2P3/CNRS-Université, Nantes, 44307, France
}

Received: 5 July 2021 / Received in final form: 15 September 2021 / Accepted: 15 October 2021

\begin{abstract}
This article presents an assessment of fuel cycle parameter impact on waste production through the prism of vitrified container and minor actinide masses, using a scenario simulated with the CLASS code. The number of canister introduces a new focus on waste production estimation for a nuclear fleet, as it helps to set the repository size for deep geological disposal of high level waste. To evaluate the number of canisters, dedicated developments to model a simplified waste vitrification unit in the CLASS package are presented. It relies on artificial neural network estimations of decay heat, $\alpha$ radiation and mass content, for different material flow coming from reprocessing and sent to vitrification. Then, the studied scenario considers a transition from a PWRs plutonium mono-recycling fleet to a plutonium multi-recycling fleet. Vitrified waste container production is calculated as a function of different material reprocessing options. Simulations shows that up to $19 \%$ variation may be observed (in 2060) in canisters' total number depending on the different assumptions. Impact of vitrification parameters such as the size of buffer before vitrification is also analysed and the importance of mixing material coming from MOX and MIX spent fuels with material from UOX spent fuels is clearly established.
\end{abstract}

\section{Introduction}

Fuel cycle prospective studies, or nuclear scenario studies, aim at looking at different possible futures for nuclear energy. They provide quantified data on several quantities of interest, such as waste production for possible fuel cycle options. Recycling plutonium from MOX spent fuels $(\mathrm{Pu}$ and depleted U mixed oxide fuels) in Pressurised Water Reactors (PWRs) is currently studied in France, see [1]. Fuel cycle studies help to quantify the impact of this strategy on deep geological waste repository and to compare it to other plutonium management choices. Those studies are based on the modelling of the full fuel cycle evolution in order to calculate, for any time and in any facility, the isotopic composition of considered materials. In this work, simulated facilities are: fuel fabrication plants (FP), reactors, cooling pools (CP), spent fuel storages (S), separation plant or reprocessing plants (SP). Separation plants recover valuable materials from spent fuel, essentially plutonium and uranium for this work. Those are sent to the fabrication plant that models fresh fuel fabrication by mixing several extracted valuable materials. Non valuable materials are sent to the wastes in order to be vitrified.

\footnotetext{
*e-mail: lea.tillard@orano.group
}

This paper presents the development of a new simplified modelling of the vitrification plant.

Simulation outputs regarding waste management in scenario studies may be of different natures: mass of different elements such as fission products (FPs), minor actinides (MAs) or specifically americium, but also directly the number of canisters where long-lived wastes are vitrified (CSD-V). Conclusions on different scenario assessments may differ regarding numerical outputs used for fuel cycle studies. For instance, as spent fuel cooling time directly depends on the spent fuel management options, such as the spent fuel sorting for reprocessing (First In - First Out or Last In - First Out), the chosen options strongly impact ${ }^{241} \mathrm{Am}$ production due to ${ }^{241} \mathrm{Pu}$ decay and thus, also the minor actinide production, as shown in [2]. On the contrary, fission product's mass at the first order depends only on the total energy production.

Transitioning from any current fleet to a plutonium multi-recycling fleet of PWRs, leaves numerous degrees of freedom for spent fuel management. Therefore, the vitrified waste amount and the CSD-V characteristics may be optimised in investigating fuel cycle parameters. In this article, focus is put on this impact quantification, as it is one of the relevant parameters that helps to set the deep geological repository size. 
The simplified vitrification process considered consists in mixing glass frit with wastes coming from spent fuel reprocessing: essentially minor actinides and fission products. Each produced canister must comply with some design criteria, for instance chemical composition, decay heat and $\alpha$ dose integrated over time.

These data depend on the reprocessed spent fuel composition. For example, MOX spent fuel reprocessing leads to more minor actinides than UOX fuel (U oxide fuel) reprocessing, and MAs are largely responsible for the $\alpha$ dose. Consequently, both spent fuel treatments do not lead to reach the same first criterion. Mixing spent fuels for reprocessing then allow to optimise the containers' load and to minimise the CSD-V production. In simulation, this mix can be obtained by adjusting the spent fuel sorting or by introducing a vitrification tank. This tank may have for example a size limit (characterised by a given mass of reprocessed material) or a time limit (characterised by the maximum time the material stays inside the tank), but other constraints could also be considered. However, material flow inside the reprocessing unit is often dictated by new fresh fuel compositions to be loaded. Competition between several objectives of scenario studies may thus appear. In addition to CSD-V production minimisation, the rapid reduction of the MOX spent fuel stockpile may also be regarded as a target. To implement this last option, old MOX spent fuels should be reprocessed first. They are characterised by an high ${ }^{241} \mathrm{Am}$ content due to ${ }^{241} \mathrm{Pu}$ decay, at the expense of the reprocessing of newly generated multi-recycling plutonium spent fuels, containing still a significant ${ }^{241} \mathrm{Pu}$ quantity. Thus, a rapid decrease of MOX stockpile may imply the production of CSD-V with high $\alpha$ radiation. Fuel cycle simulations enable to quantify those effects and perform multi-criteria optimisation.

This article aims at identifying the main relevant fuel cycle parameters for container production, and at quantifying its sensitivity to the different vitrification variables. Reactors parameters are known to be influential on the waste production, specially reactors burn-ups, but are set aside of investigations for this work. This study examines a single scenario, inspired from [1], which describes the transition from the current French fleet to a plutonium multi-recycling fleet of PWRs using the MIX $(\mathrm{Pu}$ and enriched U mixed oxide fuels) technology, described in [3]. Fuel cycle simulations are performed with the CLASS package, see [4], which was modified to add a simplified vitrification model. This new unit relies on physical models allowing the numerical estimation of decay heats, $\alpha$ doses and the mass of fission products and actinides, needed for the calculation of canister production.

The first part of this paper describes the new models, based on neural networks trained on dedicated databases built with the SMURE package, see [5]. The second part presents the CLASS developments carried out for the integration of the new simplified vitrification unit. The third and the fourth parts are dedicated to the scenario modelling with the CLASS code and the results concerning the canister production. Two main discussions are proposed: the first one develops the influence of spent fuel management, and more specifically the reprocessing priorities, while the second one focuses on canister production sensitivity to vitrification parameters.

\section{Vitrified canister modelling}

The canister production related to waste vitrification depends on several properties of the materials to be vitrified. In this work, only three global quantities are considered: the decay heat released at the canister production time, the total number of $\alpha$ decay over 10000 years per glass gram and the weight proportion of fission products and actinides in the canister. These data are not directly available in CLASS simulations as these focus on actinide inventories in each unit of the simulated nuclear fleet. To resolve that, new databases, dedicated to post-irradiation data, are built to calculate those three characteristics for material coming from reprocessed spent fuels.

This section presents how the SMURE package, see [5], is used to produce data for the following PWR fuels:

- UOX and ERU (Enriched Reprocessed U oxide fuel) respectively for enriched natural uranium oxide and enriched reprocessed uranium oxide,

- MOX and MIX respectively for plutonium oxide coming from reprocessed UOX spent fuels mixed homogeneously with depleted uranium oxide and multi-recycled plutonium oxide (from UOX, MOX or MIX reprocessed spent fuels) mixed homogeneously with enriched uranium oxide.

For each type of fuel, initial composition, discharged burn-up and cooling time are sampled to calculate the relevant values for the canister production calculation. Neural networks are then trained on these databases to build predictors used for the simplified vitrification plant modelling in CLASS. First, the fuel depletion simulations are presented, then the sampling is justified and the databases are analysed. Finally, artificial neural network precisions are assessed.

\subsection{SMURE calculations for spent fuel characterisation}

Only PWR assemblies depletion simulations are considered in this study. All assemblies are supposed to be homogeneous, meaning that the fuel composition is the same over all pin, e.g. without any plutonium content zoning as current MOX fuels (in those assemblies, 3 different plutonium contents are used to smooth the UOX/MOX interfaces, see [6]). This hypothesis should not bring strong biases neither on actinide inventories nor on fission products compositions at discharge as shown in [7]. The geometry is coming from [8] and the main data are presented in Table 1. Within the SMURE package, SERPENT2, see [9], is used as solver of the Boltzmann's equation coupled with a fourth order Runge\&Kutta solver for the depletion calculation. 16 time steps are considered for SERPENT2 calculations: 20 days, 40 days, 100 days, and then every 100 days. It covers fuel discharged burn-up 
Table 1. Main characteristics of the simulated assembly.

\begin{tabular}{lll}
\hline Parameter & Unit & Value \\
\hline Fuel pin radius & $\mathrm{cm}$ & 0.409 \\
Cladding thickness & $\mathrm{cm}$ & 0.057 \\
Pin to pin distance & $\mathrm{cm}$ & 1.26 \\
Inner tube guide radius & $\mathrm{cm}$ & 0.561 \\
Mean fuel temperature & $\mathrm{K}$ & 900 \\
Mean moderator temperature & $\mathrm{K}$ & 570 \\
Mean boron concentration & $\mathrm{ppm}$ & 450 \\
Inter-assembly gap & $\mathrm{cm}$ & 0.25 \\
Water density & $\mathrm{g} \mathrm{cm}^{-3}$ & 0.736 \\
Fuel density & $\mathrm{g} \mathrm{cm}^{-3}$ & 10.04 \\
Mean specific power & $\mathrm{W} \mathrm{cm}^{-3}$ & 330 \\
\hline
\end{tabular}

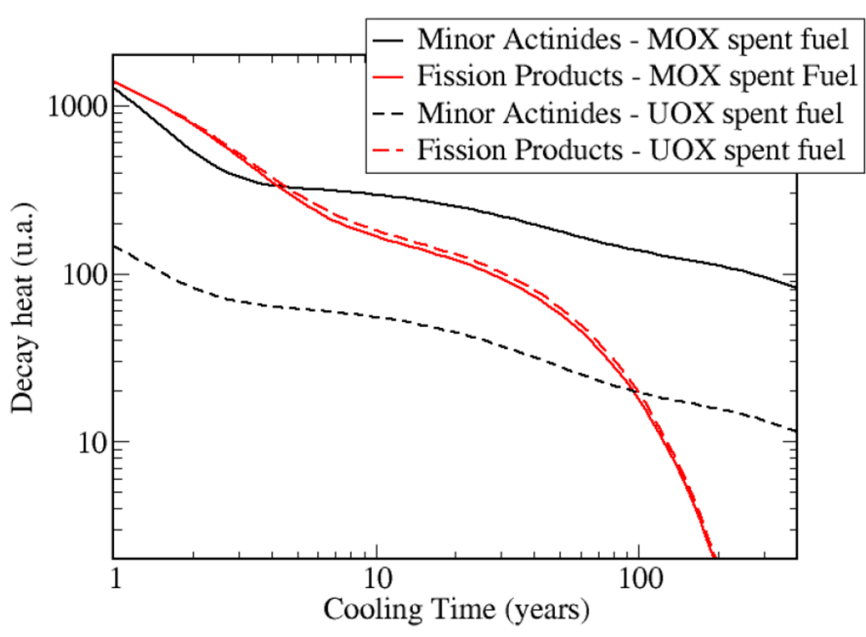

Fig. 1. Decay heat evolution after uranium and plutonium extraction.

from 0 to $55 \mathrm{GW} \mathrm{d/t.} \mathrm{ENDFB7.1} \mathrm{library} \mathrm{is} \mathrm{used} \mathrm{for} \mathrm{the}$ nuclear data.

After the depletion calculations, post-irradiation data may also be calculated with SMURE. For instance, contributions of fission products and minor actinides to the decay heat of typical UOX and MOX spent fuels are presented in Figure $1 \alpha$ activities of such spent fuels are presented in Figure 2.

Vitrification modelling must not consider the total spent fuel to evaluate post-irradiation data as chemical separation is performed to recover plutonium and uranium. Very little data of industrial chemical separation efficiencies are available in the literature and for this paper, some approximations have been done. Considered separation efficiencies are presented in Table 2. Note that, the chosen separation efficiencies are lower than typical efficiencies achieved in industrial plant which may affect the estimated canister production.

Within these hypotheses, a large number of depletion simulations are performed to build the databases containing many different spent fuel compositions, which are needed in this study.

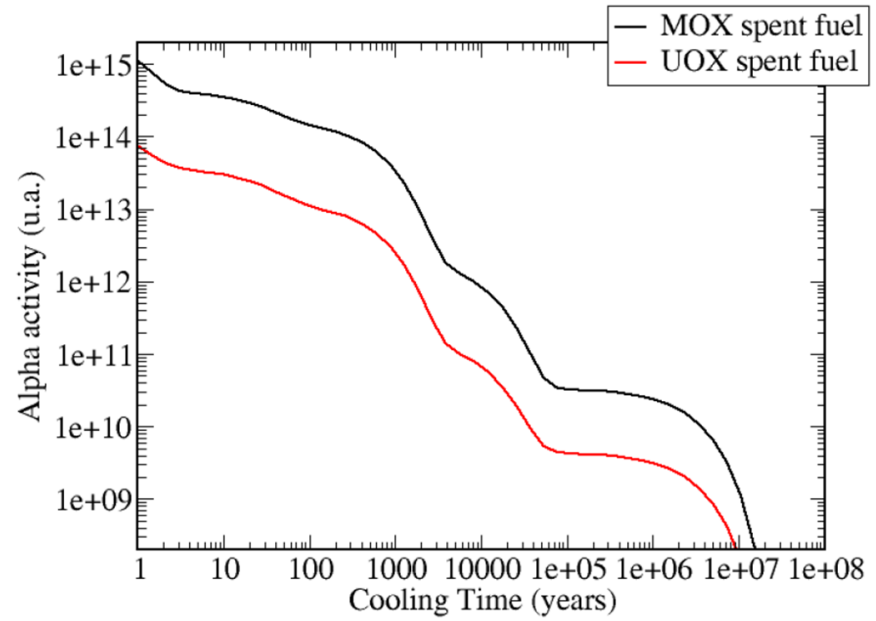

Fig. 2. Alpha activity evolution after uranium and plutonium extraction.

Table 2. Chemical separation efficiencies considered for this work.

\begin{tabular}{ll}
\hline Element & Separation efficiency \\
\hline Uranium & $99 \%$ \\
Plutonium & $99 \%$ \\
Rare gas & $100 \%$ \\
Halogen & $99 \%$ \\
Other elements & $0 \%$ \\
\hline
\end{tabular}

\subsection{Database construction}

To be flexible, only two databases are built: one for UOX and ERU fuel, one for MOX and MIX fuel; for each database calculation options are shared. The plutonium content and the enrichment are sampled as presented in Table 3. The sampling space is deliberately very large to ensure good accuracy of neural network predictions. Indeed, as those are interpolators, all the possibilities that may be simulated in further scenario studies have to be inside the training space. For this work, the reprocessing of very old UOX spent fuels as well as MIX fuels with a low cooling time are considered. Intervals for plutonium isotopic composition were inspired from [10].

Respectively, 200 and 500 initial compositions for UOX/ERU and MOX/MIX databases are identified with the Latin Hyper-cube Sampling technique. This might be considered statistically low, however precision on surrogate models are more than satisfying as shown in Section 2.3. These composition depletions are simulated with SMURE for 16 different burn-ups. Then, four cooling times are randomly sampled for the calculation of post-irradiation data. Post-irradiation data, i.e. masses, decay heat and integrated $\alpha$ dose over 10000 years, are calculated for 100 tons of spent fuel reprocessing. Those cooling times represent the elapsed time between the reactor discharged and the vitrification process.

Those values should be compared to different limits for each canister. Once again, literature may seem little 
Table 3. Sample domain for database simulations.

\begin{tabular}{lllll}
\hline & \multicolumn{2}{c}{ UOX/ERU } & \multicolumn{2}{c}{ MOX/MIX } \\
\hline Parameter & Min & Max & Min & Max \\
${ }^{234} \mathrm{U} /{ }^{235} \mathrm{U}$ & $4 \times 10^{-6}$ & $1 \times 10^{-3}$ & \multicolumn{2}{c}{-} \\
${ }^{236} \mathrm{U} /{ }^{235} \mathrm{U}$ & $3 \times 10^{-3}$ & 0.75 & \multicolumn{2}{c}{-} \\
${ }^{\mathrm{Enrichment}}$ & $3.5 \%$ & $6 \%$ & $0.1 \%$ & $5 \%$ \\
${ }^{238} \mathrm{Pu}$ & & - & $0.05 \%$ & $8 \%$ \\
${ }^{240} \mathrm{Pu}$ & & - & $8 \%$ & $45 \%$ \\
${ }^{241} \mathrm{Pu}$ & & - & $0 \%$ & $25 \%$ \\
${ }^{242} \mathrm{Pu}$ & & - & $0.5 \%$ & $25 \%$ \\
${ }^{241} \mathrm{Am}$ & - & $0 \%$ & $5 \%$ \\
Plutonium content & & - & $5 \%$ & $12 \%$ \\
Burn-up (GW d/t) & 0 & 55 & 0 & 55 \\
Cooling Time (years) & 3 & 100 & 3 & 100 \\
\hline
\end{tabular}

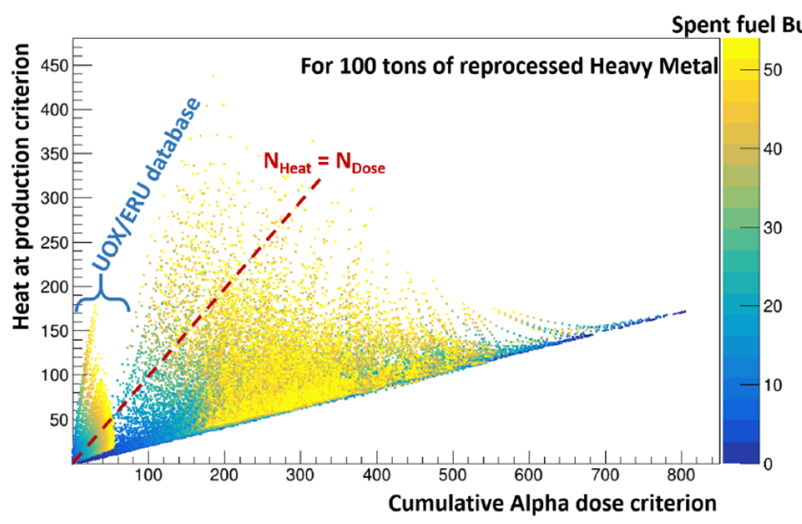

Fig. 3. Number of produced canister, regarding only decay heat or only alpha dose, while reprocessing 100 tons of heavy metal coming from different spent fuels as a function of the reactor burn-up (in GW d/t).

detailed about glass specification for the canister production. It has been chosen to take as limits, $3 \mathrm{~kW}$ at production time per canister, $2.5 \times 10^{19} \alpha$ disintegrations over 10000 years per gram of glass and a limit of $18.5 \mathrm{wt} . \%$ for the total weight proportion of nuclear wastes (fission products and actinides: FPA) in each canister.

For each criterion, the number of canister is deduced by the increased ratio of the SMURE specific quantity and the chosen limit value. Figure 3 represents the canister production, while reprocessing 100 tons of all spent fuel compositions, calculated in both databases (coloured by the discharged burn-up) regarding two criteria: the heat at canister production and the integrated $\alpha$ dose over 10000 years.

In this figure, two different behaviours may be attributed to the UOX/ERU data and the MOX/MIX data. The dots related to the UOX/ERU data are indicated on the plot. Regarding those, decay heat is the main limit, besides, after several years of cooling, the mass criterion is always dominant for canister production, as shown in Figure 4 that presents the number of canister produced by the reprocessing of 100 tons of UOX/ERU regarding mass and heat criteria.

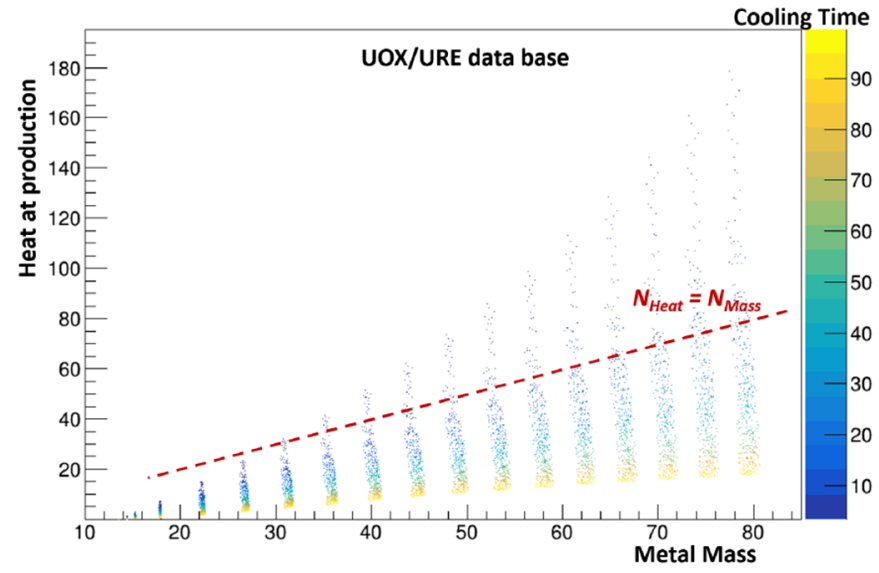

Fig. 4. Number of produced canister, regarding only decay heat or only mass, while reprocessing 100 tons of heavy metal coming from different spent UOX/ERU fuels as a function of the cooling time (in years).

In the MIX/MOX data, there is a competition between decay heat and cumulative $\alpha$ dose, depending on reactor's burn-up, plutonium isotopic composition and spent fuel cooling time. The higher the cooling time is, the higher the ${ }^{241} \mathrm{Am}$ inventory is, and therefore, the higher the cumulative $\alpha$ dose becomes, while the lower the decay heat becomes. From the MOX/MIX database, it should be noticed that cumulative $\alpha$ dose is always the sizing criterion as soon as the cooling time exceeds 15 years. For lower cooling time, no strong correlations are observed.

Finally, as the first limit reached is different for uranium fuel and plutonium fuel, this CSD-V production estimation, using the databases, suggests that some mix of UOX/ERU and MOX/MIX spent fuels in fuel cycle simulations can reduce the total amount of canister produced by optimising the containers' load.

Here, it is worth noting that those calculations are based on several hypotheses, which strongly limit the assessment of canister productions, specially the assembly depletion calculations and the chemical separation efficiencies (see Tab. 2). However, this work aims at studying, for different trajectories, the sensitivity of produced high-level wastes to fuel cycle parameters, specially the spent fuel management described by the reprocessing and fresh fuel fabrication priority order.

Using both databases, neural networks are trained to predict the three criteria of interest (FPA mass, decay heat and cumulative $\alpha$ dose), the number of produced CSD-V being directly deduced from those values during CLASS simulations.

\subsection{Model validation}

Neural networks are built for both databases. Their inputs are the fresh fuel (uranium and plutonium if needed) isotopic composition, the burn-up and the cooling time before vitrification. They allow, for each fuel type, the calculation of initial decay heat, cumulative $\alpha$ dose over 10000 years and FPA mass to be incorporated in glasses, from 100 tons of heavy metal before irradiation. 
Table 4. Criterion prediction for the 3 limits considered for canister production regarding the burn-up of the MOX or MIX spent fuels coming from the independent database built for neural network testing.

\begin{tabular}{llll}
\hline & $\begin{array}{l}\text { Cumulative } \\
\alpha \text { dose }\end{array}$ & $\begin{array}{l}\text { Heat at } \\
\text { production }\end{array}$ & $\begin{array}{l}\text { Metal } \\
\text { mass }\end{array}$ \\
\hline All BU & $2.31 \%$ & $2.51 \%$ & $0.47 \%$ \\
$\mathrm{BU}>20 \mathrm{GW} \mathrm{d} / \mathrm{t}$ & $1.38 \%$ & $1.13 \%$ & $0.20 \%$ \\
$\mathrm{BU}>33 \mathrm{GW} \mathrm{d} / \mathrm{t}$ & $1.31 \%$ & $1.10 \%$ & $0.18 \%$ \\
\hline
\end{tabular}

The predictor accuracies are here estimated by comparing the predicted values with a new set of data, completely independent from the training set. Verification is shown here for the predictors dedicated to the MOX/MIX database. In fact, the sampling space is wider than the one used for the UOX/ERU database, consequently these last predictors are more accurate and their precision's are not presented here. However, the uncertainty of those have been estimated to $0.9 \%$ for the cumulative $\alpha$ dose, $1.0 \%$ for the heat and smaller than $0.1 \%$ for the metal mass.

160 new compositions are randomly sampled over the same space (see Tab. 3) to build the training set. Each of them were used for SMURE depletion simulations and post-irradiation data are calculated for two random cooling times, leading to a set of 2590 new data for metal mass, decay heat and cumulative $\alpha$ dose of material left after separation.

This new independent MOX/MIX database is compared with the artificial neural network estimations, which results are presented in Table 4 for different burn-ups (BU). Clearly, the accuracy depends on the discharged burn-up, and the prediction may be uncertain (up to several percent) for low burn-up values. However, it would be of little interest to massively reprocess and vitrify material coming from spent fuels that may be reloaded in reactors (with discharged burn-up lower than $20 \mathrm{GW} \mathrm{d/t}$ ). Somehow, it might happen in very limited cases, for example for incomplete irradiation and long intermediate shut-down where the reactor start-up is postponed after refuelling due to some incidents.

Nevertheless, a huge majority of spent fuel in scenario studies reaches burn-up higher than $33 \mathrm{GWd} / \mathrm{t}$. In that case, neural networks' induced biases are typically lower than $1.5 \%$. The distribution of calculated biases between neural network and reference calculation are shown in Figure 5 .

Both distributions are slightly shifted from the origin and present a small bias. Looking closely, it seems that those distributions have two different behaviours. It may be because neural networks are built on a database describing two different fuel types. However, the neural networks' precision seems much better than all the uncertainties that must be coped with this approach, e.g. the chemical separation efficiencies or the depletion simulation options. Those latest may bring several percent of biases in plutonium or minor actinide inventories at the reactor

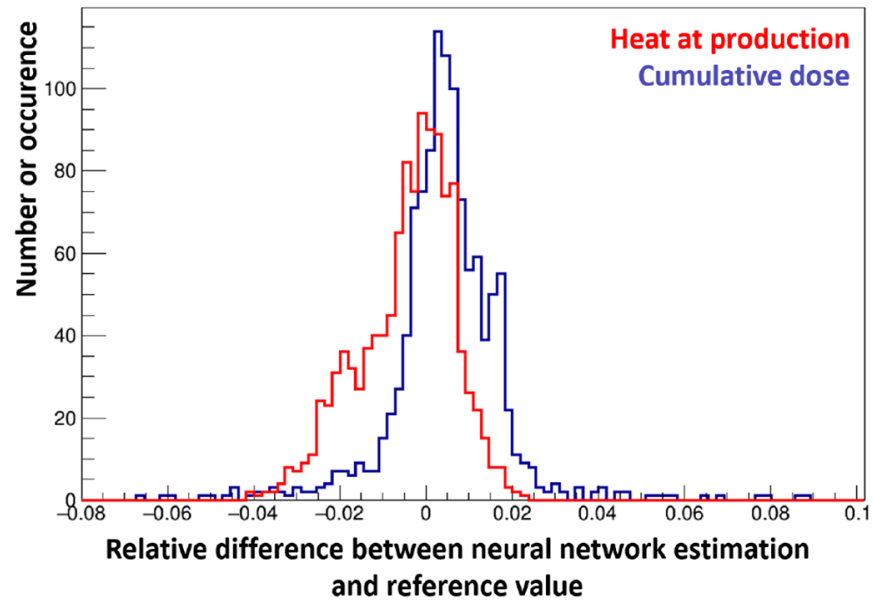

Fig. 5. Precision of decay heat at production and cumulative alpha dose over 10000 years estimation for spent fuel burn-up higher than $30 \mathrm{GWd} / \mathrm{t}$.

discharged as shown in [11]. It is also important to note that the neural network accuracy is directly the canister production precision regarding each limit. Thus, with this model, the CSD-V production uncertainty due to the use of neural networks' models is approximately $1.5 \%$.

Some other non-linear regressions could be used and the data-base could be fed with new data to increase the accuracy of the model, but a precision smaller than $1.5 \%$ seemed more than enough for this study purpose.

\section{Integration of canister production estimation in the CLASS code}

The previous section shows how meta-models can be used to calculate some of the physical properties needed for the canister production estimation during vitrification. They are implemented in the CLASS package and some developments are made to model a new type of unit: a simplified vitrification plant taking materials from reprocessing plants and evaluating the number of CSD-V produced.

\subsection{CLASS presentation and specificities}

CLASS, presented in [4], is developed since 2012 by the CNRS/IN2P3 with an initial financial support of IRSN. It models a full nuclear fleet with reactors, cooling pools, spent fuels' storages, reprocessing and fabrication plants.

In CLASS, fuel fabrication is performed on demand. It means that for each reactor loading, the fabrication plant takes, from a dedicated intermediate storage, the fissile mass needed for the fresh fuel production. This needed mass depends on the available fissile isotopic quality and the parameters of the reactor to be loaded, such as burnup and partial refuelling options. Storages are fed with different spent fuels after decay in the appropriate cooling pools. The fuel management sets the sorting of the different materials inside the storage. Thus, a fresh fuel can be 
built with recycled material coming from different reactor or fuel types if those are stored in the same storage.

The chemical separation of plutonium and uranium from the spent fuel to be reprocessed can be performed in the fresh fuel fabrication plant or in a dedicated separation plant upstream. In both cases, there is no way to identify the type of spent fuel used for the fabrication, nor its burn-up, only by its composition during chemical separation. However, both information are required, as well as the fresh fuel isotopic composition, by the neural network used in the modelling of the simplified vitrification process.

A new $\mathrm{C}++$ object (called Reactor Fuel) that gathers all these information, has been developed. The vitrification process takes place after spent fuel reprocessing that can be modelled in CLASS either in the Fabrication Plant or in the Separation Plant depending on the simulation options. At each reactor loading, a Reactor Fuel is created, storing the real discharged burn-up, the loading time, and the initial composition. Reactor Fuel characteristics are updated, at each transfer from reactors to pools and from pools to stocks until the spent fuel is sent for reprocessing. Physical characteristics of materials to be vitrified can be then estimated thanks to the information stored in the Reactor Fuel that feed the model presented in the previous section.

\subsection{Simplified vitrification plant model}

To feed recycled fuel fabrication, chemical separation is simulated and all unwanted materials are sent to wastes. Vitrification considers all the Reactor Fuels that have been reprocessed and calculates the post-irradiation data for each extracted material. Those may wait in dedicated tanks before vitrification. Two different limits for material hold up are implemented to trigger the vitrification process: a total mass limit or an additional minimum waiting time. In these cases, vitrification may occur every time the mass limit or the minimum waiting period is reached.

Those buffers for vitrification allow the mix of material coming from different spent fuels to optimise the produced vitrified canister number. Hence, the vitrification process considers the sum of the different heats, $\alpha$ cumulative doses or metal masses over all considered Reactor Fuel. Consequently, for each of those three criteria, the vitrification unit calculates the number of produced canister $N_{c}$, as expressed in equation (1), where $X_{c}$ represents the physical quantity for criterion $c$ of material coming from each Reactor Fuel and $L_{c}$ represents the chosen limit value for $c$ per canister.

$$
N_{c}=\text { floor }\left(\frac{\sum_{\text {ReactorFuel }} X_{c}}{L_{c}}\right)+1 .
$$

For each criterion, $N_{c}$ is stored for physical analysis, but the maximum value of these three criteria is kept as the final number of canisters.

Limit values, criterion definition and canister weight are left as free parameters that can be tuned for each scenario simulation. In the following, only the three aforementioned criteria are considered, but other may be implemented by analysts, if necessary.

\section{Scenario presentation}

To illustrate the new vitrification features developed in CLASS, a scenario analysing the waste container production in a PWR fleet multi-recycling plutonium is simulated. PWRs are firstly loaded with UOX and MOX fuels and gradually, as new Evolutionary Power Reactors (EPRs) are deployed, MIX fuels are introduced to multirecycle plutonium in EPR, substituting MOX fuel. In this scenario, the plutonium content of MIX fresh fuel is set to a given value and constant whatever the isotopic quality and the reactor burn-up, but the ${ }^{235} \mathrm{U}$ enrichment is adjusted as a function of the reactor constraints.

The fleet evolution described in this work is inspired from the trajectory named Scenario 8\% MIX EPR without uranium recycling into ERU fuel taken from [1], where the first EPR loaded with UOX fuel starts in 2018. This trajectory presents similarities with the French nuclear timeframe of reactor deployment for the past decades and some prospective studies. However, adaptations have been made for CLASS simulations and are presented in this section. To investigate the sensitivity of the canister production, several fuel cycle parameters related to the reprocessing strategy choices and the CLASS simplified vitrification process are sampled.

\subsection{Scenario transcription in CLASS}

The studied scenario covers the period from 1975 to 2090. All reactors are individually simulated, their commissioning and shut-down calendar and their features, such as burn-up or type of fuel, are kept constant in all trajectories.

From 1975 to 2018 the scenario uses a preexisting modelling of the French fleet including timeframe of Gen 2 PWR deployment, reactor characteristics, UOX and MOX fuel data [12]. Then, EPR deployment are extrapolated from [1] to be consistent with this paper published in 2018. 30\% MOX EPRs are operated approximately for a decade. After 2045, EPRs fuelled with 100\% MIX fuel start to increase their electricity production gradually to reach $40 \%$ of the total production, replacing the plutonium mono-recycling in MOX fuel. Once an equilibrium is reached, the plutonium consumption in 14 MIX EPRs compensates the plutonium production in 24 UOX EPRs.

EPRs are Gen $3+$ PWR producing $1530 \mathrm{MW}_{e}$ with a load factor of $83 \%$. Their core mass is 129 tons of heavy metal (HM). The fuel is refilled per third and is irradiated up to $51.8 \mathrm{GWd} / \mathrm{t}$. The fuel loading model and the irradiation model in CLASS of such reactors are the one describes in [13]. The simulated fleet evolution is shown on Figure 6.

Limits on fuel characteristics are imposed in all trajectories: initial ${ }^{235} \mathrm{U}$ enrichment maximum value in UOX fresh fuel is $5 \%$, plutonium content in MOX fresh fuel is kept below $12 \%$, initial plutonium content in MIX fuel is 


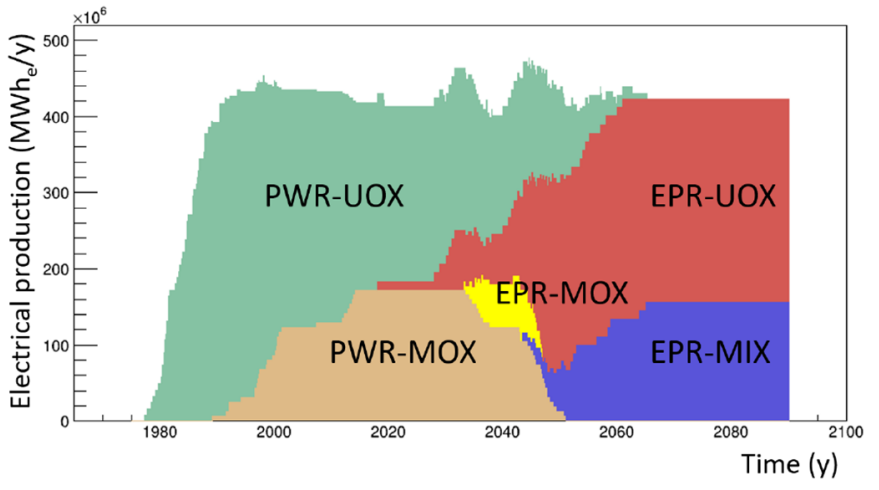

Fig. 6. Representation of the reactor deployment.

set to $8 \%$ and uranium enrichment varies to balance out the plutonium isotopic vector. No ERU fuel is considered in this scenario study.

Before 2045, only UOX spent fuels are reprocessed to feed the MOX fuel fabrication plant. After 2047, MOX and MIX spent fuels are also recycled according to reprocessing priorities defining the MIX fresh fuel production. For all fuel types, fabrication lasts 2 years, while the minimal cooling time before reprocessing is 5 years. To avoid parametrising the past, MOX fresh fuels loaded in Gen 2 PWRs are always built with a LIFO strategy, i.e. Last fuel arrived In stock First fuel Out. The fuel cycle simulated is shown on Figure 7.

Some parameters related to the CLASS vitrification modelling are kept constant for this scenario study:

- the vitrification plant, in simulations, can vitrify wastes from UOX, MOX and MIX spent fuels,

- the glass quantity per canister is $410 \mathrm{~kg}$,

- to calculate the oxide mass, the waste FPA mass is multiplied by 1.2 ,

- three limits are taken into account: the thermal power at production $(3 \mathrm{~kW} / \mathrm{CSD}-\mathrm{V})$; the cumulative alpha dose at 10000 years $\left(2.5 \cdot 10^{19}\right.$ alpha disintegration/g of glass); the FPA content (value chosen: $18.5 \mathrm{wt} . \%)$.

\subsection{Free parameters}

Fuel cycle variables taken into account for this paper are split into two categories: fleet recycling strategies and vitrification buffers.

Fleet reprocessing strategy parameters refer to:

- Stock Management strategy for MOX fuel fabrication, $\mathrm{SM}_{M O X}$ : MOX fresh fuels for EPRs are built with plutonium recovered from UOX spent fuel reprocessing with a LIFO or a FIFO, i.e. First fuel arrived In stock First fuel Out, strategy,

- Stock Management strategy for MIX fuel fabrication, $\mathrm{SM}_{M I X}$ : plutonium from UOX, MOX and MIX spent fuels feed the MIX fuel fabrication plant with a LIFO or a FIFO strategy,

- $\mathrm{P}_{X}$ : stock priority order to supply the MIX fuel fabrication plant (see $\mathrm{x}, \mathrm{y}$ and $\mathrm{z}$ on Fig. 7), either there is no priority defined and only the LIFO or
FIFO strategy is taken into account, regardless of the storage, or a priority order is defined and the LIFO and FIFO strategies apply into the stocks in a specific order. There are six possible orders (e.g. $P_{M I X}>P_{M O X}>P_{U O X}$ means that MIX spent fuels will be recycled in priority if available, then MOX spent fuels, and to complete the plutonium need UOX spent fuels).

The combination of these three parameters leads to 28 trajectories. Firstly, they are all simulated considering a direct waste vitrification, meaning that no vitrification tanks are considered to mix different materials over a given period of time. Then, simulations with vitrification buffers are considered: a 2-year or a 5-year temporal buffer and a $200 \mathrm{t}_{H M}$ or a $1000 \mathrm{t}_{H M}$ mass buffer to model vitrification tank. Those buffer values are chosen to highlight potential effects and not to describe real hold-up possibility. These additional trajectories consider either a LIFO strategy for both MOX and MIX fabrications, or a FIFO strategy, however the priority order is completely sampled.

Finally, 84 simulations are performed for this study.

\section{Results and discussions}

The scenario analysis is divided into two parts. The first one focuses on the 28 trajectories considering direct vitrification, meaning with no buffer before vitrification. This aims at analysing the strong links between the number of produced containers, the fuel cycle choices and the reprocessing strategies. Then, the second part deals with trajectories with time or mass buffers for a restraint set of fleet reprocessing strategy parameters. These trajectories are compared with the corresponding direct vitrification cases. The goal of this second part is to identify what could be the decrease of vitrified canister production with temporary material retaining.

\subsection{Reference trajectories}

In this section, only direct vitrification is considered for the analysis of container production as a function of fuel cycle options. Hence, stock management strategy for MOX and MIX fabrication and stock priority orders for MIX fresh fuel are the three variables, as summarised in Table 5.

When MIX fuel fabrication begins, in 2043, the priority for fuel type reprocessing highly impacts the reprocessed initial HM mass(in tons $t i_{H M}$ ), as depicted on Figure 8a. In 2090, maximal variation reaches $23 \%$ between $P_{M O X}>$ $P_{M I X}>P_{U O X}$ (or $\left.P_{M I X}>P_{M O X}>P_{U O X}\right)$ with a LIFO strategy for both $\mathrm{SM}_{M O X}$ and $\mathrm{SM}_{M I X}$ and $P_{U O X}>P_{M O X}>P_{M I X}\left(\right.$ or $\left.P_{M O X}>P_{U O X}>P_{M I X}\right)$ with a FIFO strategy for both $\mathrm{SM}_{M O X}$ and $\mathrm{SM}_{M I X}$. This plot shows that favouring UOX spent fuel reprocessing tends to increase the reprocessed mass (e.g. cyan lines). UOX spent fuels contain less plutonium than MOX and MIX spent fuels. As the plutonium content in MIX fresh fuels is imposed at $8 \%$ regardless of its quality, more UOX spent fuels are needed than MIX or MOX 


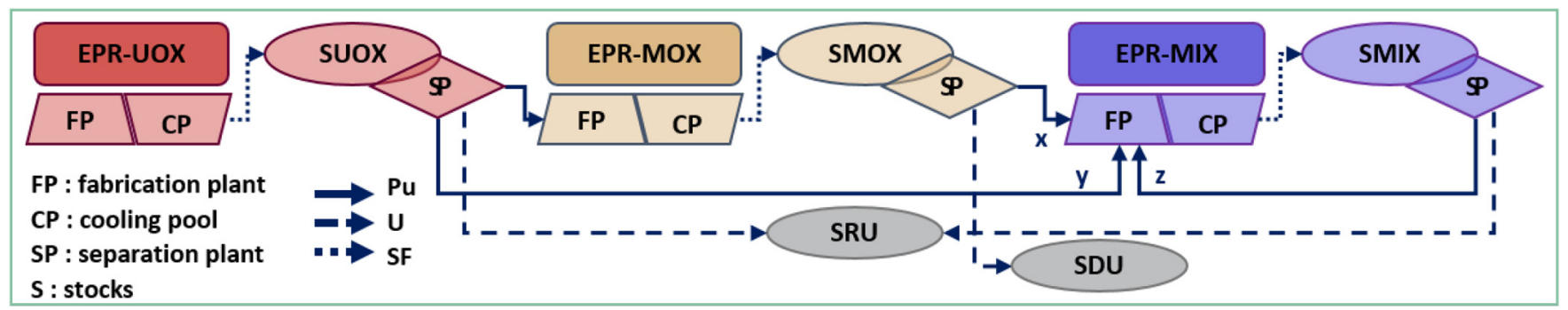

Fig. 7. Schematic representation of fuel cycle facilities; FP, CP and SP stands respectively for Fabrication Plants, Cooling Pools and Separation Plants, SUOX, SMOX and SMIX stands for Storage of different spent fuel type (respectively UOX, MOX and MIX. Finally SRU and SDU stands respectively for Stock of Reprocessed Uranium and Stock of Depleted Uranium.

Table 5. Sampled variables for the reference trajectories.

\begin{tabular}{ll}
\hline & Parameter \\
\hline $\mathrm{SM}_{M O X}$ & LIFO or FIFO \\
$\mathrm{SM}_{M I X}$ & LIFO or FIFO \\
$\mathrm{P}_{X}$ & without priority \\
& $P_{U O X}>P_{M O X}>P_{M I X}$ \\
& $P_{U O X}>P_{M I X}>P_{M O X}$ \\
& $P_{M O X}>P_{U O X}>P_{M I X}$ \\
& $P_{M O X}>P_{M I X}>P_{U O X}$ \\
& $P_{M I X}>P_{U O X}>P_{M O X}$ \\
& $P_{M I X}>P_{M O X}>P_{U O X}$ \\
Buffer & none \\
&
\end{tabular}

to recover the amount of plutonium needed for the MIX fresh fuel fabrication. However, the quality of plutonium from UOX spent fuels is much higher than the one from MOX or MIX spent fuels, the initial ${ }^{235} \mathrm{U}$ enrichments is smaller when UOX spent fuels reprocessing is prioritized. Finally, it leads to a higher plutonium consumption for the fleet. This effect is illustrated in Figure 8b, representing the total plutonium inventory which is stabilised or slightly decreasing. In 2090, maximal variation on plutonium inventory reaches $6.5 \%$ for the same extreme trajectories as for the reprocessed HM mass.

Focusing on the grey lines, where MOX spent fuel are prioritised for MIX fabrication, Figure 8a shows that the reprocessing capacity stays relatively low up to 2060 and then grows to overlap prioritised UOX spent fuel reprocessing curves. This strengthens the previous conclusion,prioritizing plutonium recovered from MOX or MIX spent fuels leads to minimize the reprocessing capacities. In fact, this sudden change corresponds to the treatment of all MOX spent fuel inventory, as visible on Figure 9a, that presents the evolution of MOX spent fuel stockpile. Then, UOX spent fuels are recycled. Figure $9 \mathrm{~b}$ shows that UOX stockpile sinks until 2075. After 2075, MIX spent fuels are also treated as UOX spent fuel amount becomes insufficient to satisfy all plutonium needs, as visible on Figure 9c. With the simulation hypotheses, especially on reactor deployments and characteristics, UOX and MIX spent fuel quantities are respectively stabilised around $3000 \mathrm{t}_{H M}$ and $5700 \mathrm{t}_{H M}$.

The impact of stock management strategy (LIFO or FIFO) on spent fuels, especially for MIX fabrication

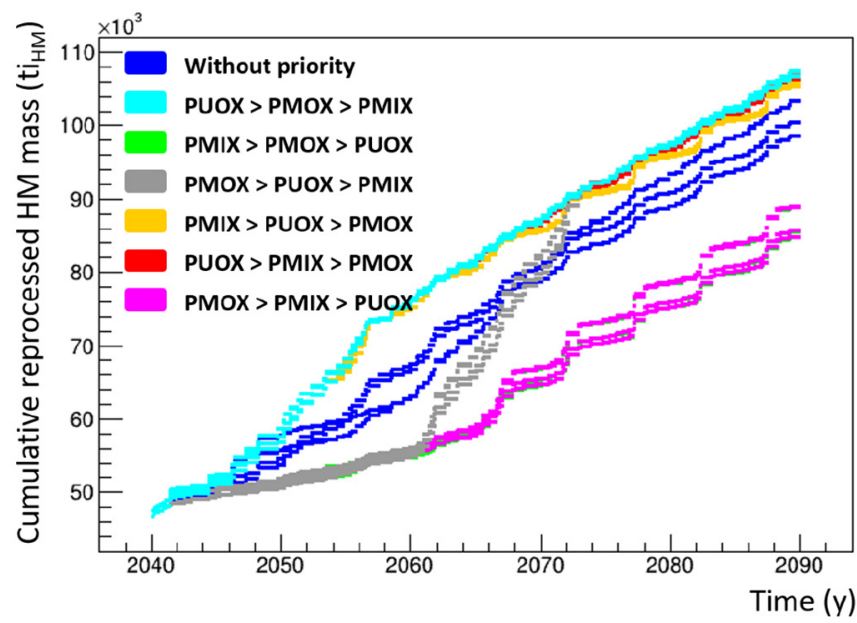

(a) Reprocessed initial Heavy Metal mass

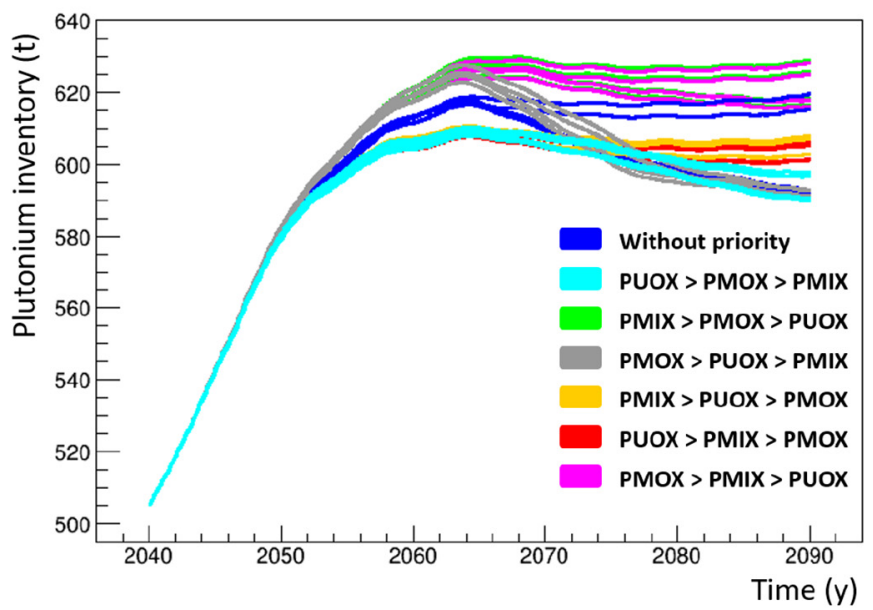

(b) Plutonium inventory

Fig. 8. Correlation between reprocessed HM mass and global plutonium inventory.

$\left(\mathrm{SM}_{M I X}\right)$, as MOX-EPRs are used for a short period, is highlighted by the no stock priority cases (blue lines in Figs. 8 and 9). In that case, materials for the fabrication plant supply are not sorted by using the spent fuel type but only by their arrival time in the different storages. When spent fuels are recycled for fresh MIX fabrication 


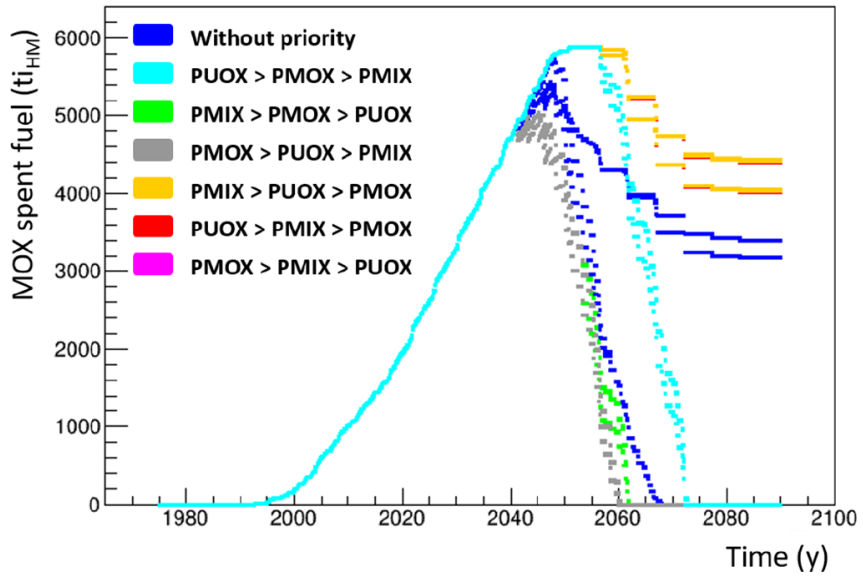

(a) MOX spent fuel

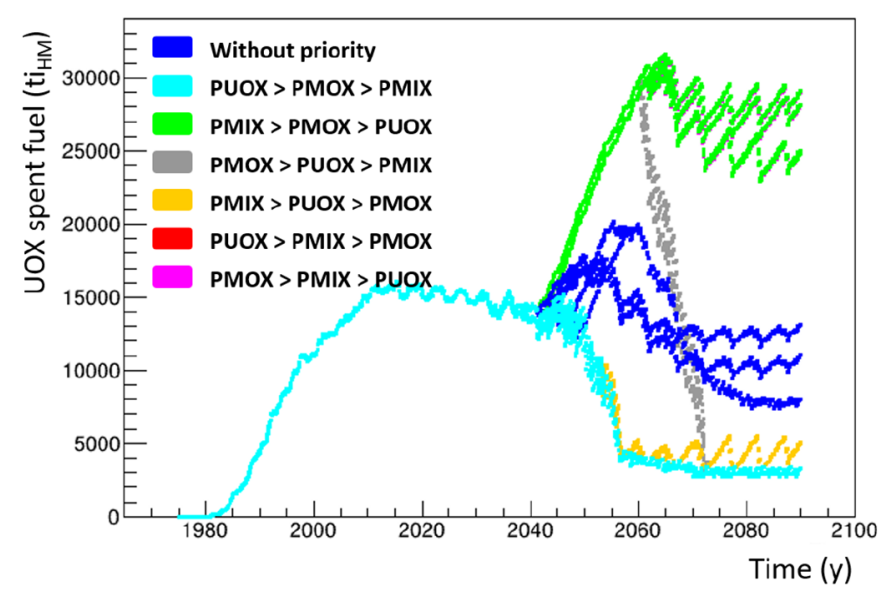

(b) UOX spent fuel

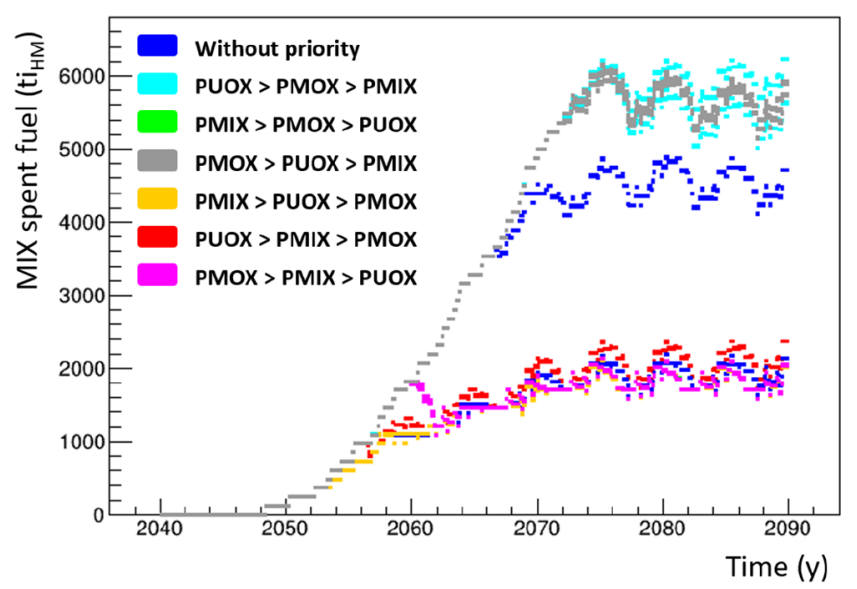

(c) MIX spent fuel

Fig. 9. Evolution of spent fuel stockpiles according to reprocessing strategies.

in a LIFO strategy, MOX spent fuel treatment is never prioritised and its stockpile is stabilised but never completely reduced. In these simulations, MOX spent fuel inventory is stabilised at approximately $3500 \mathrm{t}_{H M}$, while MIX spent fuels accumulation is stopped around $1900 \mathrm{t}_{H M}$ and UOX spent fuel quantity stabilises around $10000 \mathrm{t}_{H M}$.

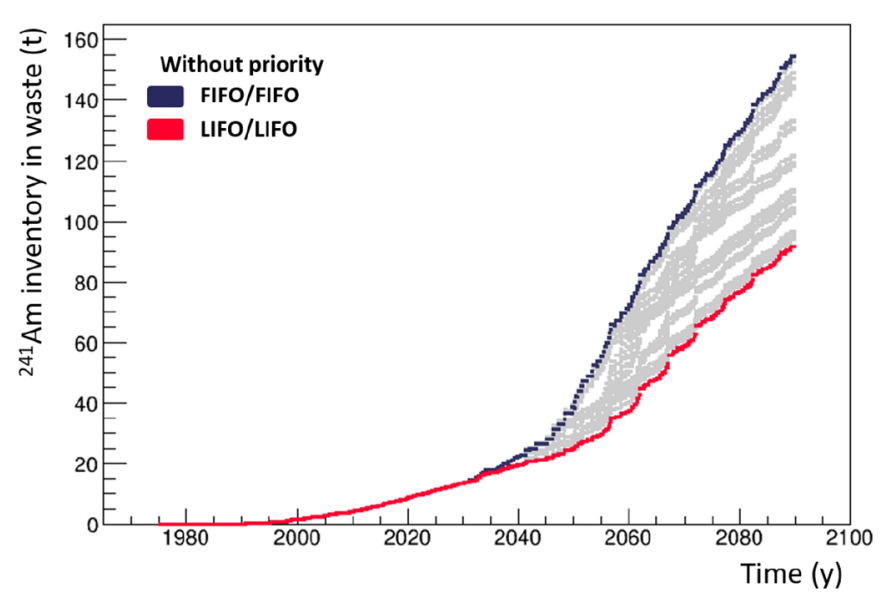

(a) ${ }^{241} \mathrm{Am}$ in wastes

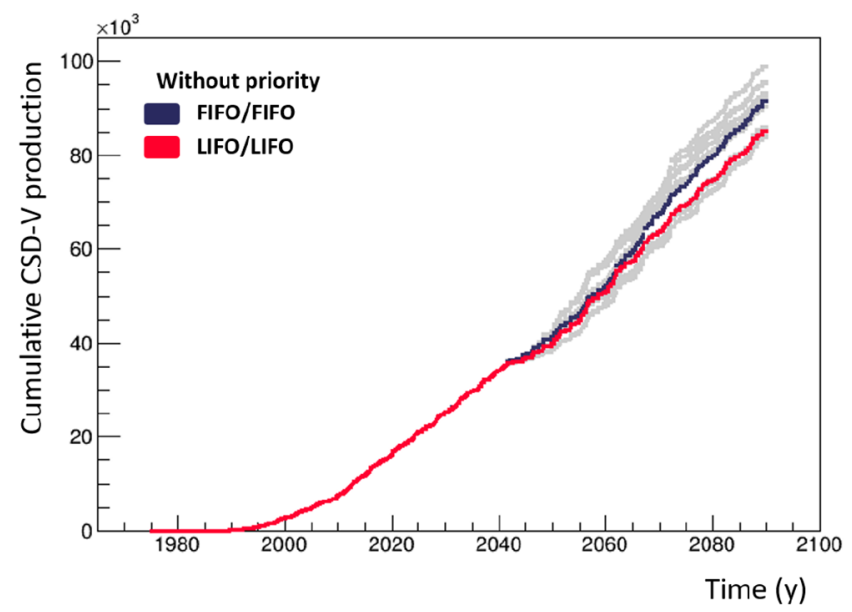

(b) CSD-V production

Fig. 10. Highlight of without priority trajectories regarding ${ }^{241} \mathrm{Am}$ inventory in wastes and CSD-V production.

The trajectory with a LIFO strategy used for MOX and MIX fabrication and no stock priority leaves some spent fuels behind for a while but it reduces the cooling time before treatment for discharged fuels. Thus, this trajectory values the most the ${ }^{241} \mathrm{Pu}$ produced in reactors and minimises ${ }^{241} \mathrm{Pu}$ decay compared to the double FIFO strategy, where long cooling time is favoured. Hence, the trajectory with a double LIFO strategy minimises the ${ }^{241} \mathrm{Am}$ inventory in wastes while the one with a double FIFO strategy maximises that quantity as depicted on Figure 10a, that presents the mass of ${ }^{241} \mathrm{Am}$ accumulated in the wastes. Same profiles are observed for americium and minor actinide inventories in wastes. However, as several criteria contribute to the CSD-V production, ${ }^{241} \mathrm{Am}$ quantity in wastes does not drive it. Decay heat coming from fission products and other actinides, as the curium contribution to the cumulative $\alpha$ dose, decreases the importance of ${ }^{241} \mathrm{Am}$ for canister production. Thus, ${ }^{241} \mathrm{Am}$ optimisation does not translate automatically in glass canister optimisation. Figure 10b highlights that, both of the extreme curves for ${ }^{241} \mathrm{Am}$ find themselves in the middle of the possible evolutions of canister production. 


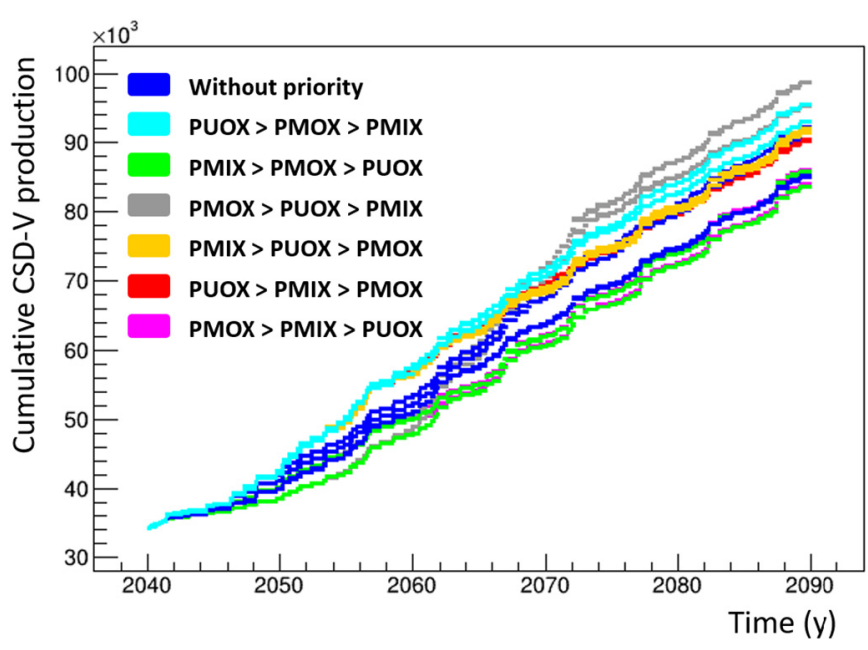

Fig. 11. CSD-V production according to reprocessing strategies.

Table 6. Maximal variations on the cumulative reprocessed HM mass and the cumulative CSD-V production over time.

\begin{tabular}{llll}
\hline & 2040 & 2060 & 2090 \\
\hline Reprocessed HM mass & $1.27 \%$ & $33.39 \%$ & $22.73 \%$ \\
Number of CSD-V & $0.03 \%$ & $18.89 \%$ & $16.75 \%$ \\
\hline
\end{tabular}

The shape of vitrified container production curves, as illustrated in Figure 11, does not look like the reprocessed heavy metal one (see Fig. 8a). These two quantities are not proportional because of different vitrification limit compensations. Lines with the lowest container production (green and pink lines) leave UOX spent fuel reprocessing in the third position, while the highest production (grey and cyan lines) is associated with MIX spent fuel treatment in the third place. Variations for the CSD-V and the HM mass between minimal and maximal trajectories are calculated in 2040, 2060 and 2090, and presented in Table 6. In 2040, reprocessing strategies do not have a major effect on the fuel cycle. In 2060, on the contrary, fuel management options greatly impact the spent fuel inventories and the spent fuel treatment. Then, in 2090, the variation decreases as storage inventories tend to an equilibrium.

Taking only the stock management strategy to build MIX fresh fuel $\left(\mathrm{SM}_{M I X}\right)$ as a parameter, differences on the number of produced canister in 2090 between LIFO and FIFO strategies are calculated for all trajectories regarding different priority orders. The maximal observed variation is equal to $7.6 \%$ and corresponds to the trajectories without priority. For the other trajectories, differences stay below $3.5 \%$. The impact of the stock management strategy as a parameter to build MOX fresh fuel $\left(\mathrm{SM}_{M O X}\right)$, is far less important, always lower than $0.2 \%$ in 2090 . This lower impact is not surprising as the MOX fuel in EPRs is used only for a decade. Nonetheless, those numbers show a relatively small impact of the stock management strategies compared to the priority parameter.
After 2075, considering that all spent fuels stocks have reached an equilibrium, the number of container produced per HM mass reprocessed varies between 1.07 CSD$\mathrm{V} / \mathrm{t}_{H M}$ and $1.15 \mathrm{CSD}-\mathrm{V} / \mathrm{t}_{H M}$ in the simulations. To understand these differences, limits reached during vitrification according to the material flow origin are assessed.

Considering the situation when only UOX spent fuels are recycled, the first limit reached is always the FPA mass, while for MOX and MIX spent fuels it is the 10000 years cumulative $\alpha$ dose limit. However, during the scenario, several spent fuel flows may be mixed during the vitrification process. The simulations show that when UOX spent fuels represent the major proportion of the reprocessed mass, the first limit reached is always the FPA mass. Then, there is a competition between decay heat and $\alpha$ dose regarding the proportion of UOX spent fuels in the mix and to the cooling time before vitrification. For example, Figure 12a shows the proportion of UOX spent fuels at each vitrification, over time, when $P_{M I X}>P_{M O X}>P_{U O X}$ and with a LIFO strategy for both MOX and MIX fuel fabrication, i.e. the trajectory minimising the canister production. For vitrification showing upper than $80 \%$ of UOX spent fuels during treatment, the FPA mass is the limit. After 2040, when the vitrification has an UOX spent fuel proportion lower than $80 \%$, the competition between thermal power and $\alpha$ dose limits is visible. For $40 \%$ to $80 \%$ of UOX, the thermal limit dominates in the LIFO strategies, as cooling time for all material is always between 5 and 6 years at vitrification step. However, in the FIFO strategies, only the alpha dose is reached below $70 \%$ of UOX spent fuel in the total reprocessed mass.

With direct vitrification dilution of MOX or MIX spent fuels with UOX is limited, a higher UOX proportion allows a clear decrease of CSD-V production, as shown on Figure 12b. In this trajectory, a sufficient mix of MIX and MOX fuels with UOX fuels may lead to a container production below $1 \mathrm{CSD}-\mathrm{V} / \mathrm{t}_{H M}$. This conclusion is further analysed in the next section as vitrification buffers are introduced.

\subsection{Mass and time buffer impacts}

Two types of buffer may be used for the vitrification modelling to introduce a hold up in the unit. In CLASS, buffers can be defined by a time period (in year) or a mass (in tons of initial heavy metal mass). For example, a temporal buffer of 2 years means that materials accumulate in the facility during 2 years of operation. Once the first batch of material has been stored in the plant for 2 years, the vitrification of all stored wastes appends. 2 years is, in that case, the maximum additional cooling time for the wastes temporarily stored in the vitrification plant. Same process can be defined for a mass hold up. The ageing of material during hold up is taken into account.

These buffers have two main effects. First, they increase the material cooling time before vitrification, decreasing in particular the associated decay heat. Then, they allow mixing material from different spent fuels before vitrification, optimising the containers' load and especially, in 


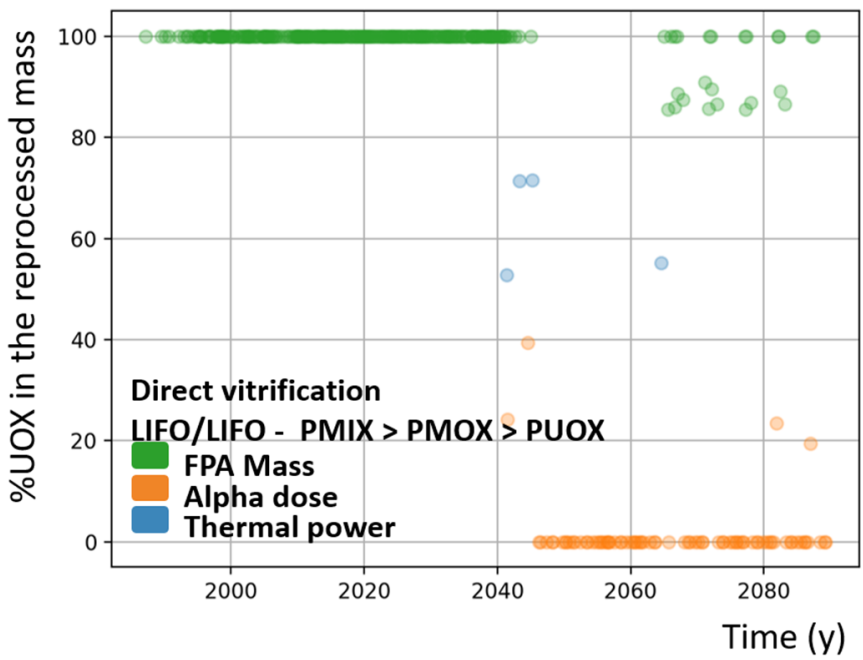

(a) Function of time

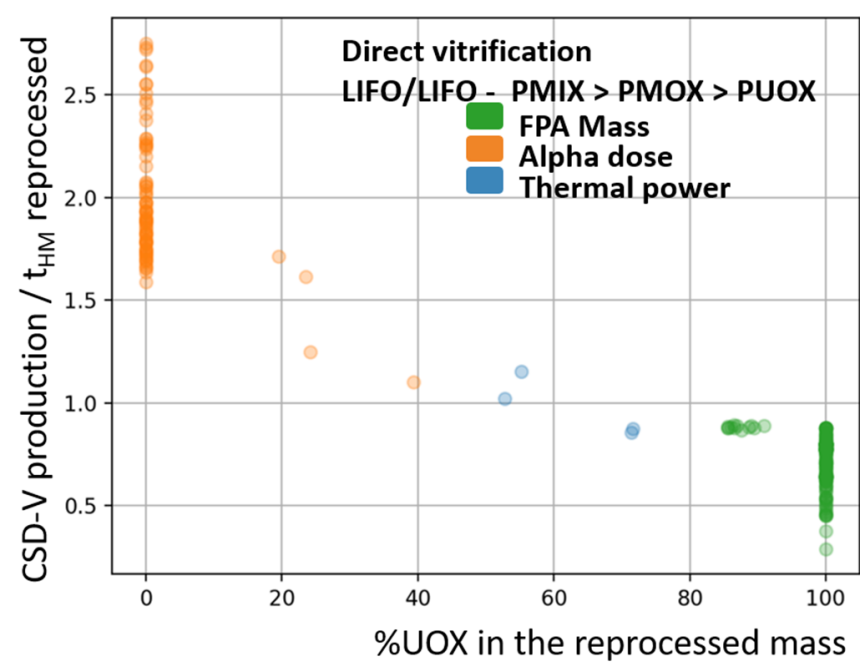

(b) Number of CSD-V

Fig. 12. Limit reached with direct vitrification according to the percentage of UOX fuel in the canister.

this scenario, decreasing the $\alpha$ dose. In the end, if the production is optimised, the chemical composition should be the major criterion.

Table 7 shows the parameters sampled in the trajectories analysed in this section.

Figure 13a shows the reduction of vitrification number due to a 5-year buffer when compared to Figure 12a; in both figures the same cycle parameters are considered. During all the simulation, vitrification occurs only four times with less than $60 \%$ of wastes coming from UOX spent fuels in the mixture. This hold up allows distinguishing more effectively the limit reached as a function of the UOX percent, as illustrated in Figure 13b. It is worth noting that a 5 years' buffer may lead to an additional cooling time up to 5 years for wastes stored in the vitrification plant. This extra cooling time is not negligible, specially with a LIFO strategy. Decay heat of fission products and actinides from UOX spent fuels decreases and
Table 7. Sampled variables for buffer trajectories.

\begin{tabular}{ll}
\hline & Parameter \\
\hline $\mathrm{SM}_{M O X} / \mathrm{SM}_{M I X}$ & LIFO/LIFO or FIFO/FIFO \\
$\mathrm{P}_{X}$ & without priority \\
& $P_{U O X}>P_{M O X}>P_{M I X}$ \\
& $P_{U O X}>P_{M I X}>P_{M O X}$ \\
& $P_{M O X}>P_{U O X}>P_{M I X}$ \\
& $P_{M O X}>P_{M I X}>P_{U O X}$ \\
& $P_{M I X}>P_{U O X}>P_{M O X}$ \\
& $P_{M I X}>P_{M O X}>P_{U O X}$ \\
& none $-2 \mathrm{y}-5 \mathrm{y}-200 \mathrm{t}-1000 \mathrm{t}$ \\
\hline
\end{tabular}

the FPA mass limit is then dominant. For MOX or MIX fuels, the cumulative $\alpha$ dose is not so sensitive to this additional cooling time, as this criterion is mainly driven by the americium 241 inventory. The FPA mass criterion dominates for major UOX presence in the mixture, then the $\alpha$ dose takes the advantage. Thus, this plot confirms that container production increases while UOX percentage in the mixture decreases. Keeping in mind that this result is bonded with the parameters and hypotheses of the simulations such as the separation efficiencies and the limit values chosen in this study, it seems that a $70 \%$ of UOX in the mixture guarantees that the chemical composition is the limit for CSD-V production. This strong role of MOX and MIX material mixing with UOX material in CSD-V production minimisation is observed for time and mass buffers.

The systematic benefit induced by vitrification tanks on CSD-V production is more important as the hold up period is longer because it amplifies both identified buffer effects. However, as the thermal power decrease does not vary linearly with the cooling time increase, see Figure 1, gain on $\mathrm{CSD}-\mathrm{V}$ production is also not proportional to the cooling time. Figure 14 represents, for three trajectories, the cumulative container production for a direct vitrification, with a 2-year and a 5-year buffer. The first trajectory chosen has the following reprocessing priority order: $P_{U O X}>P_{M O X}>P_{M I X}$, as this is associated with the maximum reprocessed HM mass (see Fig. 8a). In the opposite, the second one, $P_{M I X}>P_{M O X}>P_{U O X}$, has the lowest reprocessing rate. The cumulative number of CSD-V is also plotted for the intermediary trajectory, $P_{M O X}>P_{U O X}>P_{M I X}$, having at first a low production due to MOX spent fuel high plutonium content and after 2060, a high production as UOX spent fuels dominate the reprocessed flow. The buffer impact is stronger on the $P_{M I X}>P_{M O X}>P_{U O X}$ trajectory, as this latest is associated with the lowest UOX spent fuel reprocessing (see Fig. 9b). Indeed, in our study, the use of buffers tends to decrease the specific activity of very active wastes coming from the reprocessing of MIX and MOX spent fuels by mixing them with less active wastes coming from UOX spent fuel reprocessing. As expected, gain is smaller between 2 and 5 -year as it is between direct vitrification and 2-year buffer. A 2-year buffer is sufficient to drastically reduce the decay heat. 


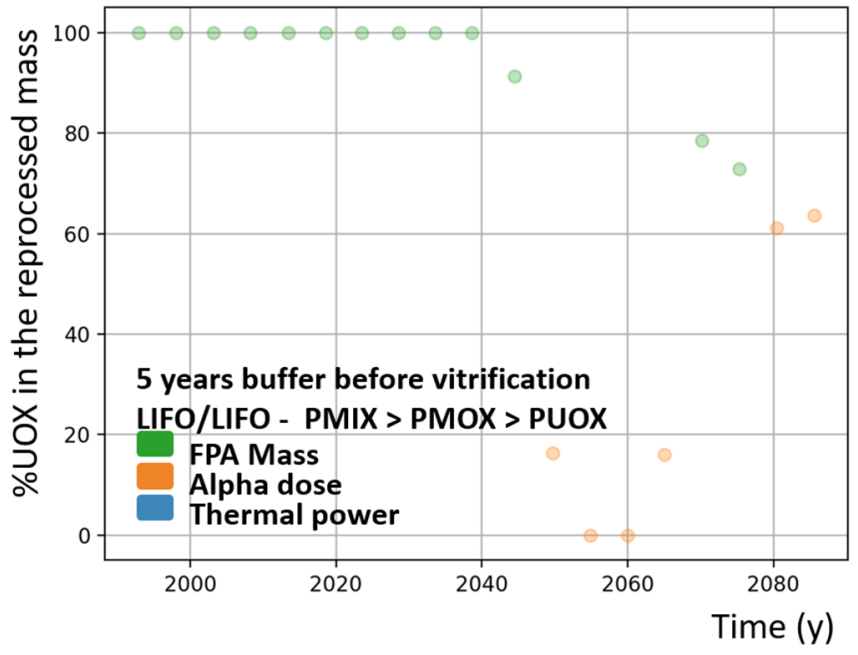

(a) Function of time

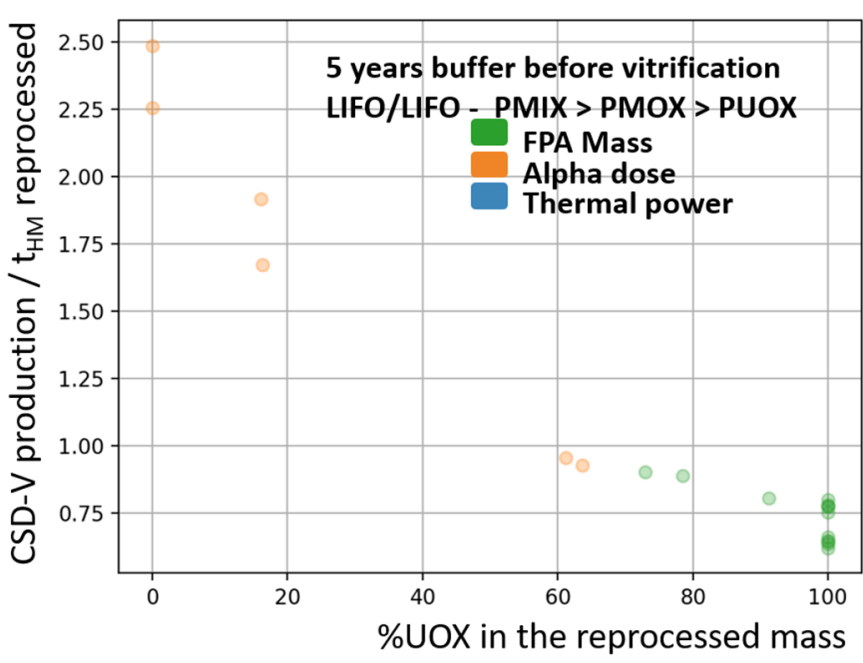

(b) Number of CSD-V

Fig. 13. Limit reached with a 5 -year buffer before vitrification according to the percentage of UOX fuel in the canister.

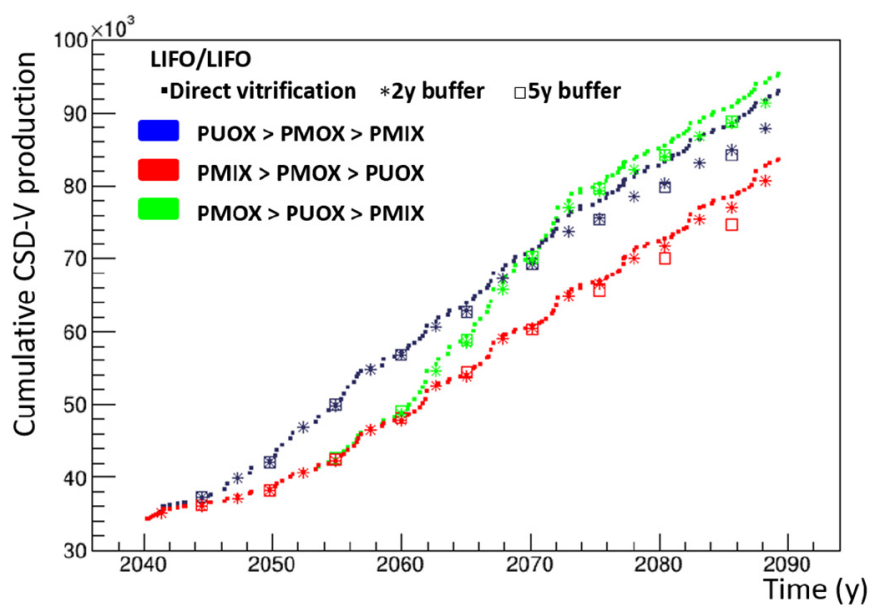

Fig. 14. Impact of time buffer.
Table 8. Variations induced in 2085.5 on the cumulative CSD-V production by buffers introduction for the three trajectories.

\begin{tabular}{lllll}
\hline & $200 \mathrm{t}$ & $1000 \mathrm{t}$ & $2 \mathrm{y}$ & $5 \mathrm{y}$ \\
\hline$P_{M O X}>P_{U O X}>P_{M I X}$ & $0.02 \%$ & $1.3 \%$ & $2.5 \%$ & $2.3 \%$ \\
$P_{U O X}>P_{M O X}>P_{M I X}$ & $0.5 \%$ & $2.8 \%$ & $4.0 \%$ & $4.7 \%$ \\
$P_{M I X}>P_{M O X}>P_{U O X}$ & $0.4 \%$ & $0.6 \%$ & $8.0 \%$ & $10.8 \%$ \\
\hline
\end{tabular}

Results for mass buffers in 2085.5 are visible in Table 8 , as 2085.5 is the last time with a vitrification occurrence in the simulation for all trajectories. A 200-ton buffer has almost no impact as it corresponds approximately to two EPR cores, with the 1000-ton buffer, improvement is noticeable: for this scenario it can be assimilated to a 1-year buffer, the mean reprocessed HM mass per year being 1200 tons.

\section{Conclusion}

In this article, the new developments in the CLASS package allowing the investigation of fuel cycle parameters' impacts on vitrified waste canister production are presented. One scenario is studied to illustrate these new features. No industrial data were used for this work so the quantified number of vitrified waste containers depends on hypothesis such as chemical extraction efficiencies, vitrified waste canister properties or reactor parameters. Metal masses in particular are strongly dependent on chemical extractions.

A new unit for simplified vitrification modelling was developed in CLASS. It relies on the estimation of decay heat, $\alpha$ activity and mass of fission products and actinides to be vitrified. These quantities depend on fuel assembly characteristics: reactor fuel type, reactor initial composition, burn-up reached at discharged and cooling time. In addition, chemical extraction efficiencies for uranium and plutonium reprocessing were chosen.

Thousands of different decay heat, $\alpha$ activity and mass estimations as a function of all these previous parameters were calculated with the SMURE package in order to build dedicated numerical databases. Artificial neural networks were then generated to estimate efficiently the three relevant criteria used in this study for the waste canister production calculation.

Once the new CLASS vitrification model developed, the investigation of how fuel cycle parameters impact the CSD-V production was performed based on a transition scenario inspired from [1]. This scenario considers a transition from a plutonium mono-recycling PWRs fleet to a plutonium multi-recycling PWRs fleet using MIX fuels. The study shows the canister production evolution as a function of some stock management strategies for fuel reprocessing, fabrication and vitrification.

Results show that the reprocessing capacities are strongly dependent on the UOX priority position for reprocessing, as UOX spent fuels contain less plutonium than MOX and MIX fuels but with a higher fissile quality. This parameter is responsible for a $23 \%$ variation of the 
reprocessed HM mass in 2090. Besides, the sampled stock management strategies induce a significant spent fuel cooling time variation before treatment and ${ }^{241} \mathrm{Am}$ production through ${ }^{241} \mathrm{Pu}$ decay is greatly impacted. Hence, a strong variation of ${ }^{241} \mathrm{Am}$ content in the waste inventory is observed, around $50 \%$ in 2090 . However, the total number of produced canister seems pretty stable and does not vary more than $17 \%$ in 2090 from a trajectory to another. As three criteria are taken into account to estimate the CSD-V production, the optimisation of ${ }^{241} \mathrm{Am}$ quantity in wastes and the total reprocessed HM mass does not translate automatically in glass canister optimisation. Thus, this study shows that the minor actinide inventory in wastes is not the first driver of the CSD-V production.

The scenario analysis shows the importance of mixing different materials from different spent fuels as it optimised the containers' load especially, for plutonium multi-recycling fuels, decreasing the $\alpha$ dose. Low radiations, decay heat and mainly $\alpha$ decays, of UOX spent fuels tend to decrease the total number of produced CSD$\mathrm{V}$ if those are treated with MOX or MIX spent fuels. The CSD-V production per ton of reprocessed spent fuel may, according to all the hypotheses, vary from less than $1 \mathrm{CSD}-\mathrm{V} / t_{H M}$ to more than $2 \mathrm{CSD}-\mathrm{V} / t_{H M}$. This observation is even more effective when buffers, representing a vitrification tank, are simulated, as they increase the material cooling time and mixing possibilities. In this study, a 2 -year buffer may offer a decrease up to $8 \%$ in the production of canisters, also depending on the stock priority.

A more precise CSD-V production optimisation would need further work, specially on the validity of reactor depletion data-bases, more realistic extraction efficiencies and canister specifications. However, this study, based on a scenario of the literature, highlights the capacity of the CLASS package to efficiently explore and analyse fuel cycle option impacts on the CSD-V production.

\section{Author contribution statement}

All the authors were involved in the preparation of the manuscript. All the authors have read and approved the final manuscript.

\section{References}

1. G. Martin, M. Guyot, F. Laugier, G. Senentz, G. Krivtchik, B. Carlier, D. Lecarpentier, F. Descamps, C. Chabert, R. Eschbach, French scenarios toward fast plutonium multirecycling in $\mathrm{PWR}$, in $I C A P P$, Charlotte, North Carolina, 2018

2. N. Thiollière, J.-B. Clavel, F. Courtin, X. Doligez, M. Ernoult, A.-A. Zakari-Issoufou, G. Krivtchik, B. Leniau, B. Mouginot, A. Bidaud, S. David, V. Lebrin, C. Perigois,
Y. Richet, A. Somaini, A methodology for performing sensitivity analysis in dynamic fuel cycle simulation studies applied to a PWR fleet simulated with the CLASS tool, EPJ Nucl. Sci. Technol. 4, 13 (2018)

3. G. Youinou, A. Vasile, "Plutonium Multirecycling in Standard PWRs Loaded with Evolutionary Fuels," Nuclear Science and Engineering - NUCL SCI ENG, vol. 151, pp. 25-45, 092005

4. B. Mouginot, B. Leniau, N. Thiollière, M. Ernoult, S. David, X. Doligez, A. Bidaud, O. Meplan, R. Montesanto, G. Bellot, et al., "Core library for advanced scenario simulation, CLASS: principle \& application," in International Conference "The Role of Reactor Physics toward a Sustainable Future" (PHYSOR 2014), pp. 12-p, 2014

5. O. Méplan, "MURE 2 : SMURE, Serpent-MCNP Utility for Reactor Evolution User Guide." http://lpsc.in2p3.fr/ MURE/html/SMURE/UserGuide/UserGuide.html, 2021

6. R. Largenton, Modélisation du comportement effectif $d u$ combustible MOX par une analyse micro-mécanique en champs de transformation non uniformes. $\mathrm{PhD}$ thesis, Université de Provence Aix-Marseille, 2012

7. U. Mertyurek, I. C. Gauld, "Development of ORIGEN libraries for mixed oxide (MOX) fuel assembly designs," Nuclear Engineering and Design, vol. 297, pp. 220-230, 2016

8. B. Rearden, W. Marshall, C. Perfetti, J. Miss, Y. Richet, "Effect of Undersampling Biases in Monte Carlo Reaction Rate Tallies," EGAMCT meeting, Kurchatov Institute, Moscow, 2013.

9. J. Leppänen, M. Pusa, T. Viitanen, V. Valtavirta, T. Kaltiaisenaho, "The Serpent Monte Carlo code: Status, development and applications in 2013," Annals of Nuclear Energy, vol. 82, pp. 142-150, 2015. Joint International Conference on Supercomputing in Nuclear Applications and Monte Carlo 2013, SNA + MC 2013. Pluri- and Trans-disciplinarity, Towards New Modeling and Numerical Simulation Paradigms

10. F. Courtin, N. Thiollière, X. Doligez, M. Ernoult, B. Leniau, J. Liang, B. Mouginot, A.-A. Zakari-Issoufou, "Assessment of plutonium inventory management in the french nuclear fleet with the fuel cycle simulator CLASS," Nuclear Engineering and Design, vol. 377, p. 111042, 042021

11. A. Somaini, S. David, X. Doligez, A. Zakari-Issoufou, A. Bidaud, N. Cappelan, O. Méplan, A. Nuttin, P. Prévot, F. Courtin, et al., "The impact of reactor model simplification for fuel evolution: A bias quantification for fuel cycle dynamic simulations," in 2016 International Congress on Advances in Nuclear Power Plants (ICAPP 2016), pp. 1045-1053, 2016

12. F. Courtin, Étude de l'incinération du plutonium en REP MOX sur support d'uranium enrichi avec le code de simulation dynamique du cycle CLASS. PhD thesis, Université Bretagne Loire, 2017

13. B. Leniau, B. Mouginot, N. Thiollière, X. Doligez, A. Bidaud, F. Courtin, M. Ernoult, S. David, "A neural network approach for burn-up calculation and its application to the dynamic fuel cycle code CLASS," Annals of Nuclear Energy, vol. 81, 072015

Cite this article as: Léa Tillard, Xavier Doligez, Gérald Senentz, Marc Ernoult, Jiali Liang, Nicolas Thiolliére, Estimation of the vitrified canister production for a PWR fleet with the CLASS code, EPJ Nuclear Sci. Technol. 7, 21 (2021) 